\title{
Efeitos de temperatura em Cromatografia Líquida de Alta Eficiência (HPLC)
}

\author{
Fernando M. Lanças \\ Instituto de Química de São Carlos, Universidade de São Paulo - USP, \\ Cep 13560-970, São Carlos, SP, Brasil \\ e-mail: flancas@iqsc.usp.br
}

Resumo

Ao longo de várias décadas, a principal característica que diferenciava claramente a Cromatografia Gasosa (GC) da Cromatografia Líquida (LC) era o fato de que a separação em GC usualmente ocorria em temperaturas elevadas, enquanto que a LC ocorria à temperatura ambiente. Entretanto, com o desenvolvimento das colunas quimicamente ligadas (ex. RP-18), mecanismos de separação mais dependentes da temperatura começaram a ser empregados e o controle de temperatura para assegurar a precisão dos tempos de retenção passou a ser utilizado também em LC. Mais recentemente, verificou-se que o uso de temperatura mais elevada em LC conduz a uma série de vantagens quando comparado às separações efetuadas à temperatura ambiente, resultando em um nicho denominado de Cromatografia Líquida a Elevadas Temperaturas (ETLC, "Elevated Temperature Liquid Chromatography") ou Cromatografia Líquida em Altas Temperaturas (HTLC, "High Temperature Liquid Chromatography"). O presente trabalho apresenta uma visão geral a respeito do papel da temperatura em cromatografia líquida, especialmente em relação a sua influência na seletividade e retenção.

Palavras-chave

Cromatografia Líquida a Altas Temperaturas; HTLC; Cromatografia Líquida em Temperaturas Elevadas; ETLC; Efeitos da temperatura em HPLC.

\section{Temperature Effects in High Performance Liquid Chromatography (HPLC)}

\section{Abstract}

For several decades the major characteristics to clearly diferentiate gas chromatography (GC) from liquid chromatography (LC) has been the fact that most GC separation occurred at elevated temperatures while LC usually has been performed at room temperature. However, upon the development of chemically bonded phases (ex. RP-18), more temperature dependent separation mechanisms started to be used, and the temperature control became an important issue to control the retention times. More recently it was observed that the use of elevated temperatures in LC broughth several advantages over the same separations performed at room temperature thus starting a new area termed ETLC (Elevated Temperature Liquid Chromatography) or HTLC (High Temperature Liquid Chromatography). This paper presents an overview on the role of the temperature in LC, in special about its influence on both selectivity and retention.

Keywords

High Temperature Liquid Chromatography; HTLC; Elevated Temperature Liquid Chromatography (ETLC); ETLC; Temperature effects in HPLC. 


\section{Introdução}

O controle da temperatura em cromatografia líquida não tem ainda recebido o merecido respeito e atenção, mesmo sabendo-se há décadas que a temperatura influencia fortemente parâmetros como solubilidade, difusividade, polaridade e viscosidade da fase móvel. Recentemente tem aumentado significantemente o número de trabalhos publicados a respeito do potencial de melhorar-se a resolução cromatográfica, através de um aumento na seletividade ou na eficiência, fatores dependentes da temperatura. Em temperaturas mais elevadas, a viscosidade é reduzida e a velocidade de difusão aumenta, fazendo com que a velocidade de transferência de massa entre a fase estacionária e a fase móvel seja aumentada e com ela a eficiência, tornando possível diminuir substancialmente o tempo de análise. O efeito da temperatura na retenção cromatográfica tem sido exaustivamente estudado recentemente, em especial sistemas baseados em fase reversa ${ }^{[1-5]}$. Apesar disto, o controle de retenção através da temperatura tem também sido estudado para outros sistemas, incluindo troca iônica, interações hidrofílicas e fase normal ${ }^{[6]}$. Mesmo considerando as vantagens apresentadas pelo uso de temperaturas elevadas em LC, um dos fatores limitantes deste enfoque é a estabilidade das fases estacionárias mais comumente empregadas.

Este trabalho discute, de forma crítica, as vantagens e limitações do uso da temperatura elevada em cromatografia líquida.

\section{Efeitos da temperatura na seletividade}

A influência da temperatura ( $\mathrm{T}$ ) no fator de capacidade (k) é usualmente expressa através da equação de Van’t Hoff (Equação 1):

$$
\ln k=-\left(\Delta H^{\circ} / R T\right)+\left(\Delta S^{\circ} / R\right)+\ln \varnothing
$$

onde: $\Delta \mathrm{H}^{\circ}=$ Entalpia do sistema; $\Delta \mathrm{S}^{\circ}=$ Entropia do sistema; $\mathrm{T}=$ Temperatura absoluta $\left({ }^{\circ} \mathrm{K}\right)$; $\mathrm{R}=$ Constante universal dos gases $\left(8,31441 \mathrm{~J} . \mathrm{K}^{-1} \mathrm{~mol}^{-1}\right) ; \mathrm{k}=$ Fator de retenção; $\varnothing=$ Razão do volume entre a fase estacionária e a móvel (também representado por $\beta$ ).

Esta relação mostra que, para uma mesma mudança na temperatura, o fator de retenção para solutos com maior $\Delta \mathrm{H}$ é mais afetado do que para solutos com menor valor de $\Delta \mathrm{H}$, fornecendo diferentes seletividades em diferentes temperaturas. Assim, uma mudança na temperatura pode permitir uma separação entre solutos, que não seria obtida empregando-se a temperatura originalmente utilizada. Entretanto, o efeito da temperatura na retenção é pequeno quando comparado com um aumento na força do solvente de eluição. Estudos com diferentes eluentes e faixas de temperatura sugerem que um aumento de $5{ }^{\circ} \mathrm{C}$ na temperatura corresponde a um aumento de $1 \%$ de acetonitrila na fase móvel em fase reversa. A relação linear existente entre k e 1/T, amplamente estudada em fases reversas empregando distintas condições, tem sido igualmente observada para separações em fase normal e até mesmo em cromatografia de troca iônica.

Apesar de que a seletividade em LC pode ser modificada através de vários parâmetros (fase estacionária, fase móvel, $\mathrm{pH}$, natureza do solvente orgânico, dentre outros), a temperatura até recentemente tem sido negligenciada como parâmetro de controle de seletividade. Com o surgimento de fornos tecnologicamente mais adequados para LC e o barateamento do seu valor, acredita-se que em breve a modificação de seletividade da separação através de modificação (geralmente aumento) da temperatura será mais popular. Deve ser lembrado que este é um dos parâmetros mais fáceis de ser ativado e controlado, usualmente através do software ou no painel do instrumento. 


\section{Temperatura $v s$. eficiência $v s$. resolução}

A resolução $\left(R_{s}\right)$ em cromatografia é comumente expressa através da equação conhecida como Equação Mestra da Resolução ("Resolution Master Equation") ou Equação de Purnell (Equação 2):

$R_{s}=\frac{\sqrt{N}}{4} \times\left(\frac{\alpha-1}{\alpha}\right) \times\left(\frac{k}{1+k}\right)$

onde: $\mathrm{R}_{\mathrm{s}}=$ resolução; $\mathrm{N}=$ número de pratos (eficiência); $\alpha=$ fator de separação $\left(\mathrm{k}_{2} / \mathrm{k}_{1}\right) ; \mathrm{k}=$ fator de retenção.

Os 3 termos da equação são interdependentes; por exemplo, pequenas mudanças em $\mathrm{k}$ geralmente ocasionam mudanças em $\alpha$ e, portanto, mudanças significativas na resolução. Uma vez que a resolução é aumentada com o aumento dos valores $\mathrm{N}$, a e $\mathrm{k}$, qualquer fator que possa alterar estes parâmetros poderá melhorar $R_{s}$. A temperatura afeta todos eles e, consequentemente, a resolução.

Yang $^{[7]}$ estudou recentemente o efeito da temperatura na eficiência das colunas $(\mathrm{N})$ operando em diferentes faixas de temperaturas, concluindo que a temperatura de análise em LC operando em fase reversa pode ser otimizada para maximizar $\mathrm{N}$. Em temperaturas mais baixas, o tempo de transferência de massas na equação de van Deemter determina a altura do prato $(\mathrm{H})$, e em temperaturas mais elevadas o termo correspondente à difusão longitudinal determina a altura do prato. Assim, operando sob velocidade linear constante, a altura do prato diminui com o aumento da temperatura em baixas temperaturas e aumenta com o aumento da temperatura em temperaturas elevadas. Outra conclusão do trabalho foi que, empregando-se elevadas temperaturas em LC, deve-se usar vazões (fluxos) elevadas da fase móvel de maneira a manter valores baixos de $\mathrm{H}$ e, consequentemente, valores elevados de $\mathrm{Ne} \mathrm{R}$.

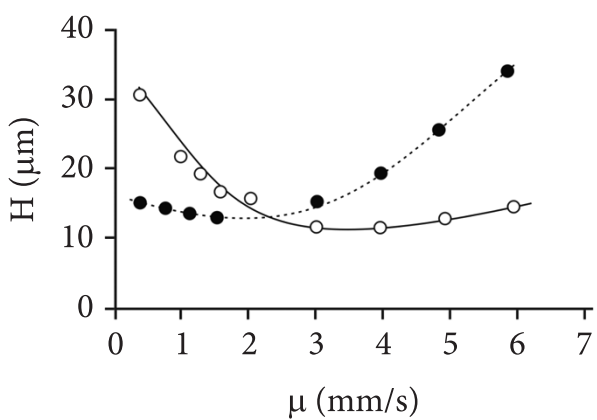

Figura 1 Variação da eficiência (medida com altura equivalente a um prato, $\mathrm{H}$ ) em função da velocidade linear media $(\mu) \cdot[\bullet]=34{ }^{\circ} \mathrm{C}[\mathrm{o}]=135^{\circ} \mathrm{C}$.

Esta relação é ilustrada na Figura 1, a qual mostra um estudo realizado variando-se a velocidade linear média da fase móvel e o valor da altura do prato resultante $\mathrm{H}$. A temperatura mais baixa (linha interrompida), valores baixos de $\mathrm{H}$, e consequentemente altos de $\mathrm{N}$, são obtidos somente em baixas velocidades lineares, até $2 \mathrm{~mm} / \mathrm{s}$. A partir desde ponto, o aumento da velocidade linear da fase móvel acarreta acelerado aumento de $\mathrm{H}$, com diminuição acentuada de N. Isto não é observado a elevadas temperaturas (linha não interrompida), em que claramente se observa que o valor de $\mathrm{H}$ se mantém praticamente inalterado a partir de $2 \mathrm{~mm} / \mathrm{s}$. Este fator tem influência na velocidade da análise, fator discutido na próxima seção.

\section{Temperatura vs. tempo de análise}

Uma das principais maneiras práticas para reduzir o tempo de análise em HPLC é o uso de colunas mais curtas. Entretanto, o comprimento da coluna é um dos principais parâmetros que afeta a eficiência da separação (Equação 3), uma vez que:

$N=\left(\frac{L}{H}\right)$ 
ou seja, o número de pratos $\mathrm{N}$ é diretamente proporcional ao comprimento da coluna $\mathrm{L}$ e, assim, alguma forma de compensação por esta perda precisa ser encontrada no caso de uma redução em L. Atualmente, uma delas é a diminuição no tamanho das partículas de fase estacionária, dp, uma vez que $\mathrm{N}$ a $1 / \mathrm{dp}$, ou seja, diminuindo-se o tamanho das partículas, aumenta-se a eficiência $(\mathrm{N})$ da coluna. Entretanto, a diminuição do tamanho das partículas, dp, apresenta como principal desvantagem um aumento significativo na pressão do sistema (Equação 4), uma vez que:

$\Delta P=\frac{\varnothing L n}{100} \cdot \frac{1}{d p}$

onde: $\Delta \mathrm{P}=$ variação da pressão na coluna (diferença entre a pressão na entrada e na saída); $\mathrm{n}$ = viscosidade da fase móvel; $\mathrm{dp}=$ diâmetro médio das partículas.

Este aumento da pressão, especialmente no caso do uso de fases estacionárias contendo partículas com $\mathrm{dp}<3 \mu \mathrm{m}$, faz com que o limite de segurança dos equipamentos de HPLC convencionais seja facilmente atingido. Em função destas variáveis, surgiu recentemente no mercado um novo enfoque para contornar estas limitações denominado U-HPLC (Cromatografia Líquida a Pressões Ultraelevadas) ou UPLC (Cromatografia Líquida com Ultradesempenho).

Por outro lado, especialmente em fase reversa, um aumento de temperatura ocasiona uma diminuição na retenção dos analitos. Além disso, a diminuição na viscosidade da fase móvel em temperaturas elevadas provoca uma diminuição na pressão do sistema, permitindo o uso de vazões (fluxos) maiores, mesmo em equipamentos "convencionais" (não U-HPLC). Assim, em temperaturas mais elevadas, torna-se possível o uso de colunas contendo partículas de diâmetro médio inferior a $3 \mu \mathrm{m}$ em equipamentos convencionais de HPLC, reduzindo substancialmente os tempos de análise.

\section{Temperatura vs. química verde}

Em LC, especialmente no modo fase reversa, o aumento de temperatura ocasiona uma redução na retenção dos analitos. Apesar dos benefícios mais óbvios serem uma redução no tempo de análise e na pressão do sistema, outro fator importante é que a quantidade de modificador orgânico na fase móvel pode ser reduzida de forma significativa. Em casos especiais, em temperaturas mais elevadas, é possível eliminar-se completamente o solvente orgânico, transformando a fase móvel em água pura. Outro fator interessante é que a redução da pressão no sistema permite o uso de etanol no lugar de metanol ou acetonitrila, amplamente usados como solventes orgânicos para fases móveis. Uma vez que o Brasil é um grande produtor de etanol, seu uso traria uma alternativa interessante quando da escassez de acetonitrila no mercado nacional, como ocorrido recentemente. Assim, os principais eluentes utilizados em fase reversa, modo mais utilizado em HPLC, passariam a ser etanol e alguns solventes não tóxicos mais baratos e de grande disponibilidade no Brasil. Este enfoque estaria em total acordo com o enfoque atual denominado química verde (green chemistry).

\section{Temperatura vs, detectabilidade}

Conforme discutido, o aumento da temperatura em LC permite uma diminuição na quantidade de solvente orgânico na fase móvel, especialmente em fase reversa. Neste caso, a fase móvel poderá ser constituída por água pura, solvente este que apresenta maior transparência na região UV, permitindo uma melhor exploração da região do espectro ao redor de $190 \mathrm{~mm}$, onde vários compostos apresentam sinal analítico, mas não são investigados por problemas de absorção dos eluentes orgânicos, ou suas impurezas, nesta região. Adicionalmente, o uso de outros 
detectores como FID (detector por ionização de chama) torna-se mais fácil e o controle de ionização em espectrometria de massas, mais preciso. Além disso, o aumento da temperatura em LC tem também demonstrado um efeito benéfico na forma dos picos, diminuindo a exigência de eluentes que, muitas vezes, requerem valores de pH e força iônica, os quais agridem as fases estacionárias empregadas nas colunas.

\section{Algumas limitações práticas do uso de temperaturas elevadas em LC}

Um dos problemas encontrados em cromatografia líquida em temperaturas elevadas é a estabilidade térmica tanto da fase estacionária quanto dos compostos a serem analisados. A maior parte das fases estacionárias utilizadas em HPLC tem como base a sílica (ex: C-18, C-8, Amino, Ciano e outras) e são estáveis até aproximadamente $60^{\circ} \mathrm{C}$; em condições excepcionais, algumas fases possuem estabilidade até próximo de $100{ }^{\circ} \mathrm{C}^{[8]}$.

O grande problema nestes casos é que o uso de água na fase móvel, associado a temperaturas elevadas, favorece a ocorrência de hidrólise e, consequentemente, perda da fase quimicamente ligada. Jones e colaboradores mostraram a existência de fases estáveis à base de sílica, as quais são estáveis até $200^{\circ} \mathrm{C}$ operando em fase reversa ${ }^{[9]}$.

A Tabela 1 ilustra as primeiras fases comercialmente disponíveis em HPLC.

Detalhes destas e de outras colunas podem ser encontrados em revisão de Yang, exclusivamente a respeito de fases estacionárias para HTLC $^{[10]}$.

A estabilidade térmica dos compostos em análise é outra preocupação com esta técnica, uma vez que serão empregadas temperaturas usualmente muito superiores às usadas em HPLC. Alguns fatores devem ser considerados, neste caso. Em geral a estabilidade térmica dos compostos obtida por outras técnicas, e em outras condições (ex. métodos térmicos), é muito maior no ambiente cromatográfico, mais inerte.

Escolhendo-se a coluna apropriada e o material de empacotamento o mais inerte possível (resíduo de metais na fase estacionária é crítico neste caso), além de colunas curtas para eluição mais rápida, estende-se bastante a estabilidade térmica das substâncias.

O uso de temperaturas elevadas em LC requer algumas modificações na instrumentação regularmente utilizada em HPLC. Uma delas é o

Tabela 1 Primeiras fases comercialmente disponíveis para cromatografia líquida a temperaturas elevadas.

\begin{tabular}{ccccc}
\hline Empresa & Material & Nome da coluna & Fase estacionária & Temp. máxima $\left({ }^{\circ} \mathrm{C}\right)$ \\
\hline Hamilton & DVB* & PRP-1 & PS-DVB & 150 \\
Jordi & DVB* & Jordi RP & DVB & 150 \\
Polymer labs & DVB* & PLRP-S & PS-DVB & 150 \\
Selerity & Sílica polidentada & Blaze-200 & C-18 & 200 \\
Shimadzu & Sílica encapsulada & Pathfinder & C18 & 200 \\
Supelco & Óxido de Zircônio & Discovery & PBD, PS & 150 \\
Supelco & Óxido de Zircônio & Discovery & Carbono C18 & 150 \\
Thermo & Carbono grafitizado & PRP-1 & Carbono grafitizado & 200 \\
\hline
\end{tabular}

* PS = Poliestireno. DVB = Divinilbenzeno. 


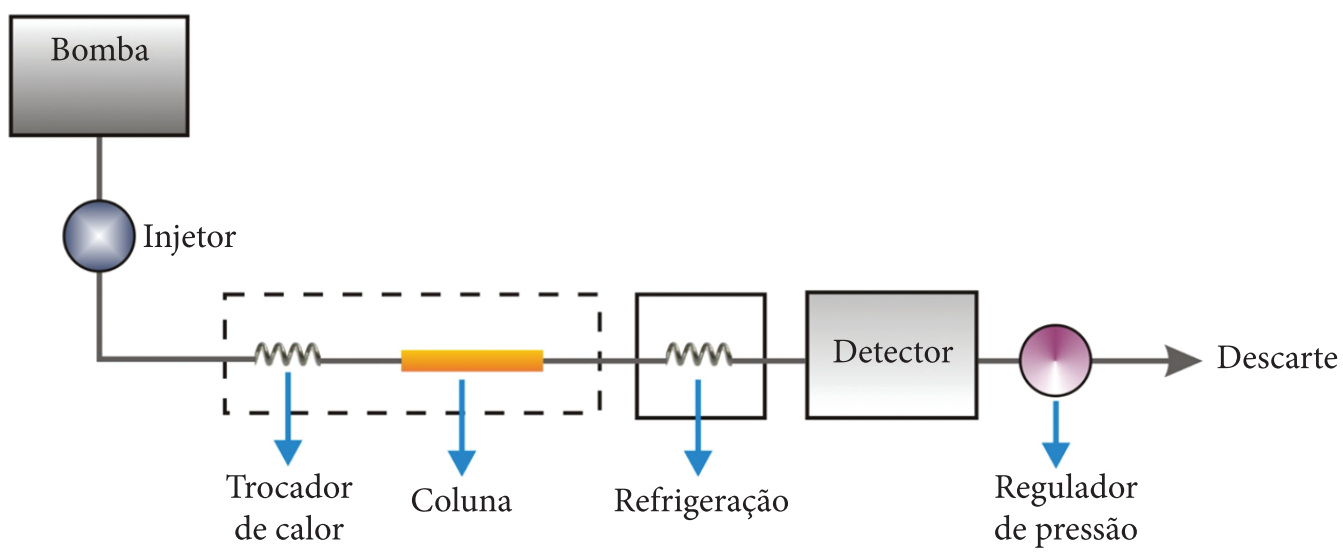

Figura 2 Instrumentação típica utilizada em Cromatografia Líquida em Alta Temperatura (HT-LC).

uso de metal, ao invés de PEEK em toda a instrumentação, e conexões sujeitas ao contacto com a temperatura elevada.

A Figura 2 ilustra um arranjo típico para HTLC.

A fase móvel é mantida no estado líquido através do regulador de pressão instalado após o detector, enquanto que um trocador de calor antes da coluna evita diferenças entre a temperatura eluente que entra no forno e quando atinge a coluna, evitando alargamento dos picos. Em algumas situações particulares, é comum instalar-se um trocador de calor antes da coluna em separações envolvendo necessidade de desnaturação.

\section{Programação de temperatura em LC}

Enquanto que em cromatografia gasosa (GC) a temperatura é a variável mais relevante, e em cromatografia com fluido supercrítico (SFC), importante, seu uso em cromatografia líquida tem sido negligenciável. Este fato tem sido atribuído a dois fatores principais: a crença sobre a baixa estabilidade dos analitos e das colunas em temperaturas elevadas. Estes argumentos já foram discutidos no item anterior, concluindo-se que a estabilidade térmica de ambos é muito superior ao que se imaginava, e que as fases estacionárias e instrumentos mais modernos operam em elevadas temperaturas sem provocar os efeitos de decomposição indesejáveis.

Considerando-se o fato de que a temperatura influência a viscosidade, solubilidade, difusividade e pressão de vapor em LC, sem dúvida ela apresenta importante contribuição na retenção, seletividade, eficiência e resolução. A partir desta constatação, vários trabalhos têm sido publicados sobre a escolha de uma temperatura ideal (isotérmica), para análises complexas por HPLC, enquanto que a otimização da separação é, geralmente, feita através do gradiente de fase móvel. Resultados mais recentes indicam que, ao invés de fixar-se a temperatura durante toda a análise, o uso de programação de temperatura permite resultados similares aos obtidos empregando-se este enfoque em cromatografia gasosa. Uma solução ainda mais interessante é a composição de programação de temperatura e de fase móvel (gradiente), o que permite a obtenção de separações melhores, em tempo menor.

Um próximo trabalho nesta série sobre os efeitos da temperatura em LC focalizará especificamente a programação de temperatura em cromatografia líquida (TPLC). 


\section{Conclusões}

O uso de temperaturas elevadas (na faixa entre 60 e $200{ }^{\circ} \mathrm{C}$ ) abre novas perspectivas para a otimização de métodos complexos em HPLC. $\mathrm{O}$ aumento da temperatura diminui a viscosidade da fase móvel, permitindo uma redução no tempo de análise sem perda de eficiência da coluna devido à melhora na transferência de massa entre a fase móvel e a fase estacionária. Como resultado, pode-se operar em velocidades lineares acima da considerada ótima à temperatura ambiente, com ganho na eficiência de separação e minimização do tempo de análise.

Como decorrência destes fatos, a programação de temperatura da fase móvel, antes restrita à cromatografia gasosa, é uma nova realidade já disponível comercialmente (apesar de que ainda pouco empregada no momento) para ser integrada no arsenal de ferramentas do usuário da HPLC.

\section{Referências}

1 Teutenberg T. Potential of high temperature liquid chromatography for the improvement of separation efficiency - A review. Analytica Chimica Acta 2009; 643:1-12. PMid:19446057. http://dx.doi. org/10.1016/j.aca.2009.04.008

2 Yang Y. Subcritical water chromatography: A green approach to high-temperature liquid chromatography.
Journal of Separation Science 2007; 30(8):11311140. PMid:17595948. http://dx.doi.org/10.1002/ jssc. 200700008

3 Teutenberg T, Tuerk J, Holzhauser M, Giegold S. Temperature stability of reversed phase and normal phase stationary phases under aqueous conditions. Journal of Separation Science 2007; 30(8):11011114. PMid:17595945. http://dx.doi.org/10.1002/ jssc. 200600507

4 Teutenberg T. High temperature liquid chromatography - A brief review about an emerging technique. Chromatography Today 2010; 3(3):3-6.

5 Zhun C, Goodall D, Wren S. Elevated temperature HPLC: principles and applications to small molecules and biomolecules. LCGC Asia Pacific 2005; 8(1):48-59.

6 Lundanes E, Greibrokk T. Temperature effects in liquid chromatography. Advances in Chromatography $2006 ; 2: 45-77$.

7 Yang Y. A model for temperature effect on column efficiency in high-temperature liquid chromatography. Analytica Chimica Acta 2006; 558:7-10. PMid:17386578. http://dx.doi.org/10.1016/j.aca.2005.11.011

8 Vanhoenacker G. Elevated temperature and temperature programming in conventional liquid chromatography - fundamentals and applications. Journal of Separation Science 2006; 29(12):1822-1835. PMid:16970186. http://dx.doi.org/10.1002/jssc.200600160

9 Jones A, Marin S, Clark J, Porter N. In: Proceedings of the XXIX International Symposium on High Performance Liquid Phase Separations and Related Techniques; 2005; Stockholm. Stockholm; 2005. p. 3-30.

10 Yang Y. Stationary Phases for LC Separations at Elevated Temperatures. LCGC North America 2006; 24(S4):53-58. 